\title{
Low Cost Touch Button of Tablet for Literacy in Developing Countries
}

\author{
Yujie $\mathrm{Li}^{\mathrm{a},{ }^{*},}$, Akira Yamawakia, Seiichi Serikawa ${ }^{\mathrm{a}}$ \\ ${ }^{a}$ Kyushu Institute of Technology \\ 1-1, Sensui, Tobata, Kitakyushu 804-8550, Japan \\ *Corresponding Author: yzyjli@126.com
}

\begin{abstract}
In recent years, with the global popularity of information technology, more and more digital mobile products have been developed. These products have led to a strong expansion of social interventions, including those that try to improve literacy of student in low-income countries. Some of the products have been called successful, but little credible evidence exists for those claims. In this paper, we present a new touch screen technique for education assistance. The main contributions of this system are as follows: (1) an energy-conserving tablet is developed and experimentally tested. (2) The low cost with best battery life. As a result, this low cost touch button of tablet can be manufactured by commonly used in poor countries.
\end{abstract}

Keywords: Tablet, touch button, circuit.

\section{Introduction}

New information technologies are of growing importance around the world. These information and communications technologies (ICTs), especially mobile devices, may have special benefits for learning, both in and out of schools. According to a report by United Nations Educational $^{(1)}$, Scientific and Cultural Organization (UNESCO), this is the first time in history that the world has more connected mobile devices than people. Despite the ubiquity of mobile technologies and their increased use in educational settings, little empirical evidence supports their use for learning. At the same time, available evidence from developing countries shows that a significant progress has been made toward international goals for education ${ }^{(1)}$.

However, in many developing countries, enrolment is upon average, and gender parity always exists. In addition, the types of educational resources found within many schools have uninvolved. The target of the Education for
All by UNESCO is very difficult to achieving. Hence, ICT applications are apparent to promote learning, even in poor schools, leading to concerns about equitable access to computers, mobile tablets, and Internet connections ${ }^{(1)}$.

There exists much ICT equipment for learning in developed countries. For example, Software Engineering Company developed an easily use tablet for children education $^{(2)}$. It has been found that the tablet is benefits for developed countries. A much cheaper and energy-conserving tablet needs to be developed for developing countries in order to meet the requirements of local infrastructure conditions ${ }^{(3)}$. In developing countries, most people still have no access to electricity. The children need to do housework for living ${ }^{(4)}$. Furthermore, most of children do not have the chance to learning.

To fact of these real problems, in this paper, we propose a cheaper and energy-conserving tablet for self-learning. This new tablet translates into several possible benefits for children education ${ }^{(5)}$. First, an energy-conserving tablet is developed and experimentally tested. It just need to charging the battery in school, can be used for a long time. Second, a flexible and arbitrary shaped touch button is designed. So, it meets the needs for learning while working. Third, the low cost with best battery life ${ }^{(6)}$. The equipment is so cheap that every child can use it. As a result, this smart tablet can be manufactured by commonly used in developing countries.

\section{Systems and Methods}

\section{$2.1 \quad$ Features}

The touch screen system contains three parts, touch buttons, LCD display, and Mbed MCU ${ }^{(5)}$. The child can set the touch buttons in any types or shapes freely. As they touch the buttons, the signal is passed to Mbed MCU. Finally, the result is shown on the LCD display. The touch 
screen is set around the arm. People can press the button, and output the signal to the display remotely. The work process is shown in Figure 1.
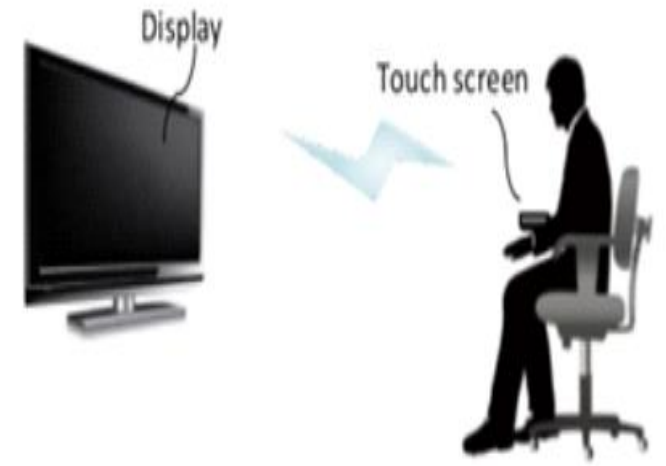

Fig. 1. Concept of the proposed tablet

\subsection{System and Circuits}

In the new touch screen system, we designed some low cost touch buttons for inputting and an energy-conserving circuit for controlling. The touch operation is received by the buttons. Then, send the signal to Mbed MCU to the communication element.

(a) Touch Button Design

We designed a touch screen as shown in Figure 2. The new touch screen contains two parts, one is the touch button part, and the other is a flexible touching LCD. The touch button is used to input the keys exactly. The flexible touching LCD with a pen is used to drawing some maps or paintings. Meanwhile, the flexible touching LCD is also used to displaying. LCD (liquid crystal display) is a thin flat display device. It consists of a number of color or monochrome pixels and Places in the square in front of the light source or reflector. LCD is thin, space-saving and compare with CRT, its energy consumption is very small. At the same time, LCD does not flicker, can reduce the monitor damage to the eyes. Because of these advantages, LCD is suitable for electronic devices with batteries ${ }^{(7)}$.

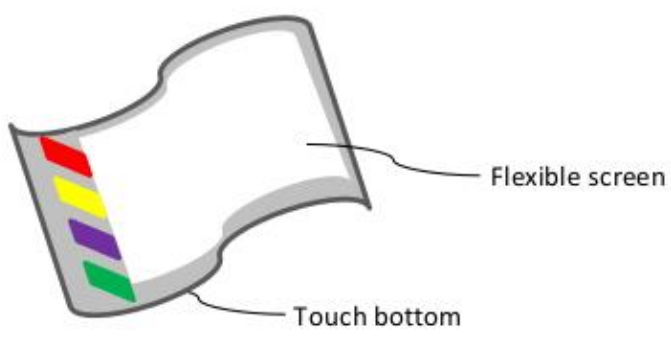

Fig. 2. Design of Flexible touch panel

Touch button actually is a touch switch. It is a very practical circuit. Touch switch is widely used in automatic control systems, such as computer, automation instrumentation, and $\mathrm{CNC}$ machine (computer numerical control machine) tools and so on ${ }^{(5)}$. In this system, the touch switch is be used and gets good results

At first, we need to design the buttons like Figure 3. It takes low-cost metal film as the button. The buttons can be cut in any shapes. Such as in order to learn the animals, the teacher just need to cut the button by the animals' shapes. So, students can easily recognize the animal and press the button to choose the answer.

We design a new circuit for this touch button. The proposed new circuit is energy conserving as shown in Figure 4 . We use $5 \mathrm{~V}$ battery, resistance $\mathrm{R} 1=10 \mathrm{M} \Omega, \mathrm{R} 2$ $=\mathrm{R} 3=\mathrm{R} 4=\mathrm{R} 5=10 \mathrm{~K} \Omega$. Transistor $\mathrm{Q} 1$ is Toshiba transistor $\mathrm{C} 1815$ and Q2 is A1015.

The electric current is about $20 \mathrm{nA} / \mathrm{s}$. So, the touch buttons are much energy-conserving than the traditional one.

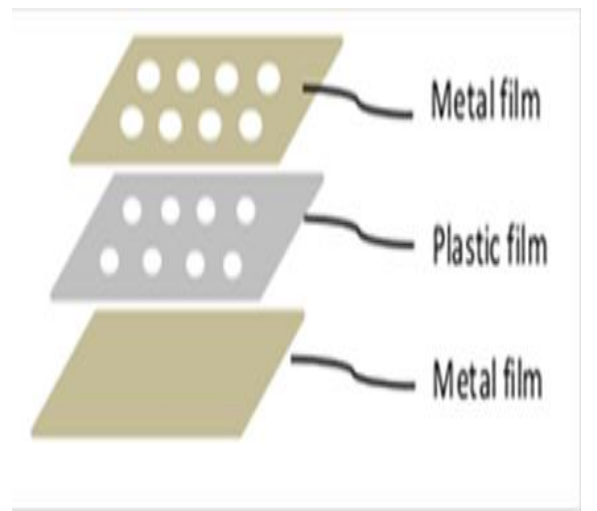

(a) The structure of flexible button

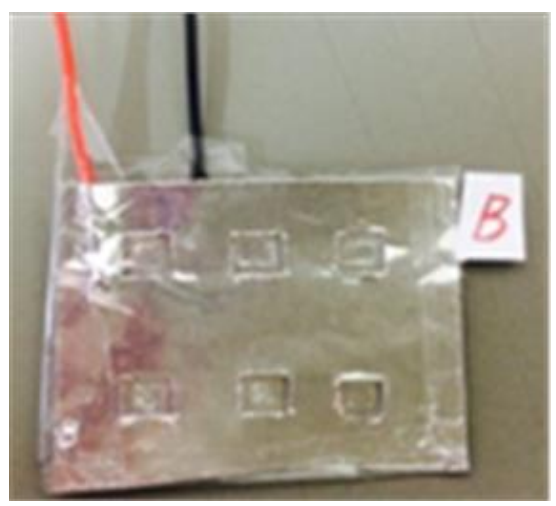

(b) The designed button "B"

Fig. 3. Touch button 


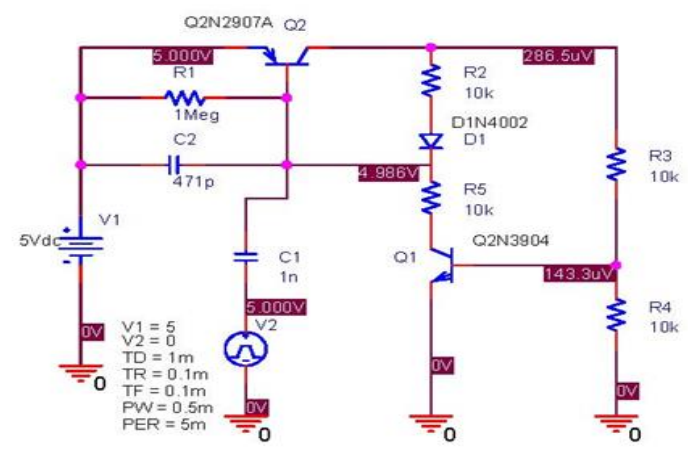

(a) The circuit of touch button

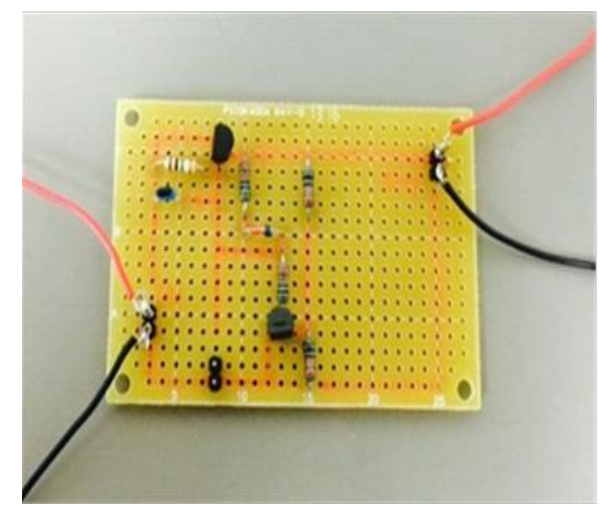

(b) The entity of circuit

Fig. 4. The circuit of system

(b) Mbed processing unit

In this system, we take Mbed NXP LPC1768 MCU for computing in Figure 5. The Mbed NXP LPC1768 Microcontroller is made for prototyping all sorts of devices; include USB, Ethernet, SD and Wifi communication.

The Mbed is based on a 32-bit ARM Cortex-M3 core with $96 \mathrm{MHz}$. It includes 512KB Flash memory, 32KB RAM and a lot of interfaces including built-in Ethernet, USB host and devices, and other interfaces. The pins (pin 5 to pin 30) can be used as Digitalln and DigitalOut interfaces. Figure 6 is the terminal layout of Mbed NXP LPC1768.

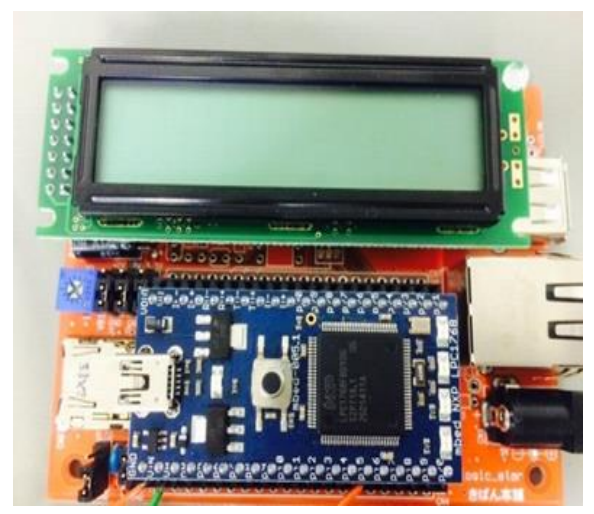

Fig. 5. Mbed smarter kit board

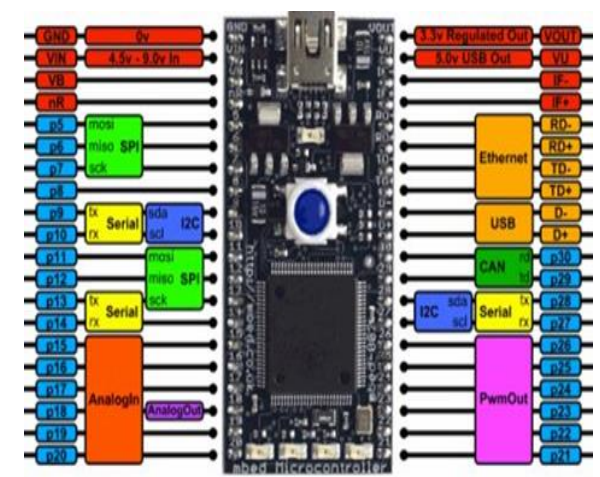

Fig. 6. Terminal Layout of Mbed microcontroller

The Mbed was powered using a $5 \mathrm{~V}$ battery, which also connect with the touch screen. If press the touch button or screen, the Mbed will be work. Pin 15 Pin 18 are set as the signal input pins. Mbed based touch screen is much cheaper than the traditional one.

\section{Results and Discussions}

In the experiment, we did some tests in our laboratory. At first, on the LCD, the question is shown, such as: the teacher asked: "Is this a rose or peony?" And then, the students press the button of the proposed touch screen and select the answer to output (see Figure 7). In our test, the answer is "this is an apple." So, the output is "A".

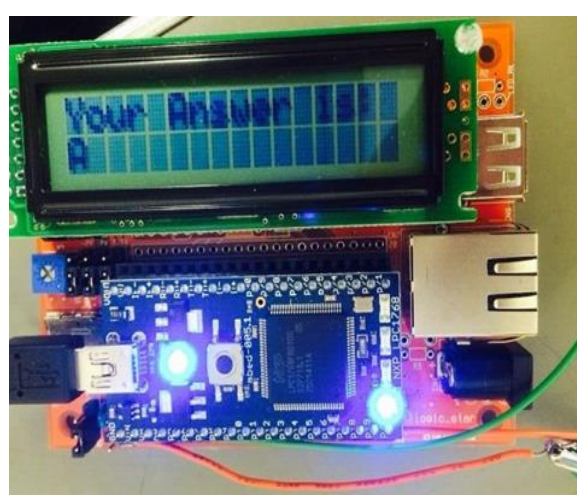

Fig. 7. Demo results after operation

\section{Conclusions}

In this paper, we present a low cost touch button for tablet for education assistance. Students in poor countries can use the proposed touch screen/button to learn or interact with teacher both in class and at home. The main contributions of this system are as follows: (1) An energy-conserving tablet is developed and experimentally tested. (2) The low cost with best battery life. As a result, this low cost touch button for tablet can be manufactured by commonly used in poor countries. In future, we will design 
a flexible touch panel and add the wireless communication module to strengthen the system.

\section{Acknowledgment}

This work is supported in part by Grant in Aid for Non-Japanese Researchers by NEC C\&C Foundation. The author also wishes to thank the anonymous reviewers and researchers for the useful comments and resources that helped improve the quality of this paper.

\section{References}

(1) United Nations Educational, Scientific and Cultural Organization: "Technology, Broadband and Education", Book, January 2013

(2) D.A. Wagner, N.M. Castillo, K.M. Murphy, M. Crofton, F.T. Zahra: "Mobiles for literacy in developing countries and effectiveness framework", Prospects, Vol.44, No.4, pp.119-132,2014

(3) Beth Beschorner, Amy Hutchison: "Ipads as literacy teching tool in early childhood", International Journal of Education in Mathematics, Science and Technology, Vol.1, pp.16-24(2013)

(4) K. Arinivasan, V. Ahukla: "Low cost tablet design: technical challenges", India Supplement, Vol.1, No.5, pp.10-11,2012

(5) C. Shen, R. Ryall, C. Forlines, A. Esenther, F.D. Vernier, K. Everitt, M. Wu, D. Wigdor, M.R. Morris, M. Hancock, E. Tse: "Informing the design of direct-touch tabletops", IEEE Computer Graphics and Applications, Vol.26, No.5, pp.36-46,2006

(6) Y. Agarwal, A. Bishop, T.B. Chan, M. Fotjik, P. Gupta, A.B. Kahng, L. Lai, P. Martin, M. Srivastava, D. Sylvester, L. Wanner, B. Zhang: "RedCooper: Hardware sensor enabled variability software testbed for lifetime energy constrained application", E-scholarship, pp.1-10,2014.

(7) H. Lu, S. Serikawa, "Design of Freely Configurable Safety Light Curtain Using Hemispherical Mirrors", IEEJ Transactions on Electrical and Electronic Engineering, vol.8, no.S1, pp.S110-S111, 2013. 